\title{
Uncertain Zero-One Law and Convergence of Uncertain Sequence
}

\author{
Zhiqiang Zhang, ${ }^{1,2}$ Weiqi Liu, ${ }^{3}$ and Xiumei Chen ${ }^{4}$ \\ ${ }^{1}$ School of Economics and Management, Shanxi University, Taiyuan 030006, China \\ ${ }^{2}$ School of Mathematics and Computer Science, Shanxi Datong University, Datong 037009, China \\ ${ }^{3}$ Institute of Management and Decision, Shanxi University, Taiyuan 030006, China \\ ${ }^{4}$ School of Information Engineering, Shandong Youth University of Political Science, Jinan 250103, China
}

Correspondence should be addressed to Zhiqiang Zhang; sjzhangzhiqiang@sxdtdx.edu.cn

Received 20 October 2015; Accepted 12 January 2016

Academic Editor: Luca Guerrini

Copyright (C) 2016 Zhiqiang Zhang et al. This is an open access article distributed under the Creative Commons Attribution License, which permits unrestricted use, distribution, and reproduction in any medium, provided the original work is properly cited.

\begin{abstract}
This paper is concerned with situations in which the uncertain measure of an event can only be zero or one, and the uncertain zeroone laws are derived within the framework of uncertainty theory that can be seen as the counterpart of Kolmogorov zero-one law and Borel-Cantelli lemma, which can be used as a tool for solving some problems concerning almost sure convergence of uncertain sequence.
\end{abstract}

\section{Introduction}

Zero-one laws are such magic results in probability theory that provide criteria for the situations in which the probability of an event can only be zero or one. A natural problem that comes to us is in what situations the uncertain measure of an event can only be zero or one, which will be investigated within the framework of uncertainty theory in this paper.

Uncertainty theory is a different type of theory from probability theory for dealing with indeterminacy. It is mainly used to deal with belief degrees, and probability theory is mainly used to deal with frequencies. Perhaps some people think that the belief degrees should be modeled by subjective probability or fuzzy set theory. However, Liu [1] demonstrated that it is usually inappropriate because both of them may lead to counterintuitive results in this case. For rationally dealing with belief degrees, uncertainty theory was founded by Liu [2]. The mathematical foundation of uncertainty theory is normality, duality, subadditivity, and product axioms of uncertain measure, which is different from probability theory. Since uncertainty theory was founded by Liu [2], many scholars devoted themselves to study of uncertainty theory, and fruitful theoretic results have been received. Liu [1] established uncertain calculus and proved the fundamental theorem. The existence and uniqueness theorem of solution of uncertain differential equation was first proved by Chen and Liu [3], and some stability theorems were proved by Yao et al. [4]. More importantly, Yao-Chen formula obtained by Yao and Chen [5] establishes a relationship between uncertain differential equations and ordinary differential equations. More materials can be found in Liu [6]. Nowadays, uncertainty theory has become a branch of axiomatic mathematics.

In real world, the degrees of belief from experts' advice about some events are frequently used by people as the basis of decisions making. Uncertainty theory is a useful tool for solving such problems since it is an efficient mathematical system to deal with belief degrees associated with human uncertainty. Nowadays, it has been successfully applied in uncertain finance; for example, European option, American option, and Asian option pricing were studied by Liu [1], Chen [7], Sun and Chen [8], and Zhang and Liu [9] within the framework of uncertainty theory, respectively. Chen and Gao [10] employed uncertain differential equation to model interest rate; following their model Zhang et al. [11] investigated the pricing problem of interest rate option. Besides, Liu et al. [12] derived the currency option pricing formula by using uncertain differential equation to model currency rate. And it 
also has been applied in many other fields, such as uncertain programming (Liu and Yao [13]), uncertain risk analysis (Liu and Ralescu [14]), uncertain optimal control (Zhu [15]), and uncertain differential game (Yang and Gao [16]).

Uncertain zero-one law is different from zero-one law in probability theory, because uncertain measure indicates the degree of belief that an event will occur, and uncertainty theory and probability theory have different operational laws. Uncertain zero-one law can be used for solving some problems concerning almost sure convergence as well as the role of Kolmogorov zero-one law and Borel-Cantelli lemma in probability theory, since it is a useful tool to deal with the problems of uncertain sequence convergence almost surely. In this paper, we will discuss in what situations the uncertain measure of an event can only be zero or one and provide the criteria for such situations within the framework of uncertainty theory. Some results of almost sure convergence of uncertain sequence are also presented in this paper.

The rest of this paper is organized as follows. In the next section, some preliminaries as needed are introduced. In Section 3, the uncertain zero-one laws are presented and some conditions for the almost sure convergence of uncertain sequence are given. Finally, we make a brief conclusion in Section 4.

\section{Preliminary}

For a better understanding of this paper, we introduce some concepts of uncertainty theory as needed in this section.

Definition 1 (see [2]). Let $\Gamma$ be a nonempty set, and let $\mathscr{L}$ be a $\sigma$-algebra over $\Gamma$. An uncertain measure is a function $\mathscr{M}$ : $\mathscr{L} \rightarrow[0,1]$ such that the following hold.

Axiom 1 (Normality Axiom). $\mathscr{M}\{\Gamma\}=1$ for the universal set $\Gamma$.

Axiom 2 (Duality Axiom). $\mathscr{M}\{\Lambda\}+\mathscr{M}\left\{\Lambda^{c}\right\}=1$ for any event $\Lambda$.

Axiom 3 (Subadditivity Axiom). For every countable sequence of events $\left\{\Lambda_{i}\right\}$ we have

$$
\mathscr{M}\left\{\bigcup_{i=1}^{\infty} \Lambda_{i}\right\} \leq \sum_{i=1}^{\infty} \mathscr{M}\left\{\Lambda_{i}\right\} .
$$

A set $\Lambda \in \mathscr{L}$ is called an event. The uncertain measure $\mathscr{M}\{\Lambda\}$ indicates the degree of belief that $\Lambda$ will occur. The triplet $(\Gamma, \mathscr{L}, \mathscr{M})$ is called an uncertainty space. In order to obtain an uncertain measure of compound event, a product uncertain measure was defined by Liu [1].

Axiom 4 (Product Axiom). Let $\left(\Gamma_{k}, \mathscr{L}_{k}, \mathscr{M}_{k}\right)$ be uncertainty spaces for $k=1,2, \ldots$. The product uncertain measure $\mathscr{M}$ is an uncertain measure on the product $\sigma$-algebra $\mathscr{L}_{1} \times \mathscr{L}_{2} \times \cdots$ satisfying

$$
\mathscr{M}\left\{\prod_{k=1}^{\infty} \Lambda_{k}\right\}=\bigwedge_{k=1}^{\infty} \mathscr{M}_{k}\left\{\Lambda_{k}\right\}
$$

where $\Lambda_{k}$ are arbitrarily chosen events from $\mathscr{L}_{k}$ for $k=1,2$, $\ldots$, respectively.

Definition 2 (see [17]). An uncertainty space $(\Gamma, \mathscr{L}, \mathscr{M})$ is called continuous if, for any events $\Lambda_{1}, \Lambda_{2}, \ldots$, one has

$$
\mathscr{M}\left\{\lim _{i \rightarrow \infty} \Lambda_{i}\right\}=\lim _{i \rightarrow \infty} \mathscr{M}\left\{\Lambda_{i}\right\}
$$

provided that $\lim _{i \rightarrow \infty} \Lambda_{i}$ exists.

Definition 3 (see [18]). The events $\Lambda_{1}, \Lambda_{2}, \ldots, \Lambda_{n}$ are said to be independent if

$$
\mathscr{M}\left\{\bigcap_{i=1}^{n} \Lambda_{i}^{*}\right\}=\bigwedge_{i=1}^{n} \mathscr{M}_{i}\left\{\Lambda_{i}^{*}\right\}
$$

where $\Lambda_{i}^{*}$ are arbitrarily chosen from $\left\{\Lambda_{i}, \Lambda_{i}^{c}, \Gamma\right\}, i=1,2$, $\ldots, n$, respectively, and $\Gamma$ is the sure event.

Definition 4 (see [2]). An uncertain variable is a measurable function from an uncertainty space $(\Gamma, \mathscr{L}, \mathscr{M})$ to the set of real numbers; that is, $\{\xi \in B\}$ is an event for any Borel set $B$.

Definition 5 (see [2]). The uncertainty distribution $\Phi$ of an uncertain variable $\xi$ is defined by

$$
\Phi(x)=\mathscr{M}\{\xi \leq x\}
$$

for any real number $x$.

Definition 6 (see [1]). The uncertain variables $\xi_{1}, \xi_{2}, \ldots, \xi_{n}$ are said to be independent if

$$
\mathscr{M}\left\{\bigcap_{i=1}^{n}\left(\xi_{i} \in B_{i}\right)\right\}=\bigwedge_{i=1}^{n} \mathscr{M}\left\{\xi_{i} \in B_{i}\right\}
$$

for any Borel sets $B_{1}, B_{2}, \ldots, B_{n}$ of real numbers.

Definition 7 (see [2]). The uncertain sequence $\left\{\xi_{i}\right\}$ is said to be convergent a.s. to $\xi$ if there exists an event $\Lambda$ with $\mathscr{M}\{\Lambda\}=1$ such that

$$
\lim _{i \rightarrow \infty}\left|\xi_{i}(\gamma)-\xi(\gamma)\right|=0
$$

for every $\gamma \in \Lambda$. In that case we write $\xi_{i} \rightarrow \xi$ almost surely (a.s.).

Definition 8 (see [2]). Let $\xi$ be an uncertain variable. Then the expected value of $\xi$ is defined by

$$
E[\xi]=\int_{0}^{+\infty} \mathscr{M}\{\xi \geq r\} d r-\int_{-\infty}^{0} \mathscr{M}\{\xi \leq r\} d r
$$

provided that at least one of the two integrals is finite.

Theorem 9 (see [2]). Let $\xi$ be an uncertain variable. Then, for any given number $r>0$, one has

$$
\mathscr{M}\{|\xi| \geq r\} \leq \frac{E[|\xi|]}{r}
$$




\section{Uncertain Zero-One Law}

Definition 10. If $(\Gamma, \mathscr{L}, \mathscr{M})$ is an uncertainty space and $T$ is a nonempty index set, sub- $\sigma$-algebras $\mathscr{G}_{t}$ of $\sigma$-algebra $\mathscr{L}, t \in T$, are termed independent if, for each $m=2,3, \ldots$, each choice of distinct $t_{i} \in T$,

$$
\mathscr{M}\left\{\bigcap_{i=1}^{m} \Lambda_{i}^{*}\right\}=\bigwedge_{i=1}^{m} \mathscr{M}\left\{\Lambda_{i}^{*}\right\},
$$

where $\Lambda_{i}^{*}$ are arbitrarily chosen from $\left\{\Lambda_{i}, \Lambda_{i}^{c}, \Gamma\right\}$ in sub- $\sigma$ algebra $\mathscr{G}_{t_{i}}$.

Definition 11. Let $\mathscr{G}$ be a collection of subsets of a set $\Gamma$. The smallest $\sigma$-algebra containing $\mathscr{G}$ is called $\sigma$-algebra generated by $\mathscr{G}$, denoted by $\sigma(\mathscr{G})$.

Definition 12. Let $\left\{X_{t}, t \in T\right\}$ be a sequence of uncertain variables, where $T$ is a nonempty index set. The smallest $\sigma$-algebra with respect to which the uncertain variable $X_{s}$ is measurable, $\forall s \in T$, is called $\sigma$-algebra generated by $\left\{X_{t}, t \in T\right\}$, denoted by $\sigma\left(X_{s}, s \in T\right)$.

Definition 13. Let $X=\left\{X_{n}, n \geq 1\right\}$ be an uncertain variable sequence and denote

$$
\mathscr{B}^{*}=\bigcap_{n=1}^{\infty} \sigma\left(X_{k}, k \geq n\right) .
$$

Then $\mathscr{B}^{*}$ is called tail $\sigma$-algebra or tail event field on $X$.

Theorem 14. Let $\left\{X_{n}, n \geq 1\right\}$ be an independent uncertain variable sequence. Then, for any event $\Lambda$ in tail $\sigma$-algebra $\mathscr{B}^{*}$, one has $\mathscr{M}\{\Lambda\}=0$ or 1 .

Proof. For any $n \geq 1$, since $\mathscr{B}^{*} \subset \sigma\left(X_{k}, k \geq n+1\right), \mathscr{B}^{*}$ is independent of $\sigma\left(X_{k}, k \leq n\right)$. Therefore, $\mathscr{B}^{*}$ is independent of $\mathscr{A}=\bigcap_{n=1}^{\infty} \sigma\left(X_{k}, k \leq n\right)$. Then $\mathscr{B}^{*}$ and $\sigma(\mathscr{A})$ are independent.

On the other hand, since $\sigma(\mathscr{A})=\sigma\left(X_{k}, k \leq 1\right) \supset \mathscr{B}^{*}$, $\mathscr{B}^{*}$ is independent of itself. For any $\Lambda \in \mathscr{B}^{*}$ we have

$$
\mathscr{M}\left\{\Lambda \cap \Lambda^{c}\right\}=\mathscr{M}\{\Lambda\} \wedge \mathscr{M}\left\{\Lambda^{c}\right\} .
$$

Therefore, $\mathscr{M}\{\Lambda\}$ is either 0 or 1 .

Corollary 15. Let $\left\{X_{n}, n \geq 1\right\}$ be an independent uncertain variable sequence and let $\mathscr{B}^{*}$ be a tail $\sigma$-algebra. Then $\mathscr{B}^{*}$ measurable uncertain variable $Y$ is degenerated; that is, $Y$ is a constant with uncertain measure 1.

Proof. For any $c \in R,(Y \leq c) \in \mathscr{B}^{*}$, we have $\mathscr{M}(Y \leq c)=0$ or 1 . Take

$$
c_{0}=\inf \{c: \mathscr{M}(Y \leq c)=1\}
$$

Then

$$
\mathscr{M}\{Y \leq c\}= \begin{cases}0, & c<c_{0} \\ 1, & c \geq c_{0} .\end{cases}
$$

Hence, whether $c_{0}$ is finite or infinite, we have $\mathscr{M}\left\{Y=c_{0}\right\}=$ 1 .
Corollary 16. Let $\left\{X_{n}, n \geq 1\right\}$ be a sequence of independent uncertain variables. Then

(i) $\limsup \sup _{n \rightarrow \infty} X_{n}$ and $\liminf _{n \rightarrow \infty} X_{n}$ are degenerated;

(ii) the uncertain measure of events such as $\{\omega$ : $\sum_{n} X_{n}$ covergences $\}$ and $\left\{\omega: \lim _{n}(1 / n) \sum_{j=1}^{n} X_{j}=0\right\}$ is 0 or 1 .

Let $\left\{\Lambda_{n}, n \geq 1\right\}$ be a sequence of events in uncertainty space $(\Gamma, \mathscr{L}, \mathscr{M})$. If $\omega \in \limsup _{n \rightarrow \infty} \Lambda_{n}=\bigcap_{n=1}^{\infty} \bigcup_{m=n}^{\infty} \Lambda_{m}$, then $\omega \in \bigcup_{m=n}^{\infty} \Lambda_{m}$ for every $n$; that is, no matter how large we choose $n$ there is always some $m \geq n$ such that $\omega \in \Lambda_{m}$ or, equivalently, $\omega \in \Lambda_{m}$ for infinitely many values of $m$ or, equivalently, for arbitrarily large values of $m$. A convenient way to express this is

$$
\omega \in\left\{\Lambda_{n} \text { i.o. }\right\}=\left\{\Lambda_{n} \text { infinitely often }\right\} .
$$

Theorem 17. (i) Let $\left\{\Lambda_{n}, n \geq 1\right\}$ be arbitrary uncertain events. If $\sum_{n=1}^{\infty} \mathscr{M}\left\{\Lambda_{n}\right\}<\infty$ holds, then

$$
\mathscr{M}\left\{\Lambda_{n} \text { i.o. }\right\}=0 .
$$

(ii) Let $\left\{\Lambda_{n}, n \geq 1\right\}$ be independent uncertain events in a continuous uncertainty space $(\Gamma, \mathscr{L}, \mathscr{M})$. If $\sum_{n=1}^{\infty} \mathscr{M}\left\{\Lambda_{n}^{c}\right\}<\infty$ holds, then

$$
\mathscr{M}\left\{\Lambda_{n} \text { i.o. }\right\}=1 \text {. }
$$

Proof. (i) We have

$$
\begin{aligned}
\mathscr{M}\left\{\Lambda_{n} \text { i.o. }\right\} & =\mathscr{M}\left\{\limsup _{n \rightarrow \infty} \Lambda_{n}\right\}=\mathscr{M}\left\{\bigcap_{n=1}^{\infty} \bigcup_{m=n}^{\infty} \Lambda_{n}\right\} \\
& \leq \mathscr{M}\left\{\bigcup_{m=n}^{\infty} \Lambda_{n}\right\} \leq \sum_{m=n}^{\infty} \mathscr{M}\left\{\Lambda_{n}\right\} \longrightarrow 0
\end{aligned}
$$$$
\text { as } n \longrightarrow \infty
$$

(ii) By continuity and independence, we have

$$
\begin{aligned}
\mathscr{M}\left\{\Lambda_{n} \text { i.o. }\right\} & =\mathscr{M}\left\{\bigcap_{n=1}^{\infty} \bigcup_{m=n}^{\infty} \Lambda_{m}\right\} \\
& =1-\mathscr{M}\left\{\bigcup_{n=1}^{\infty} \bigcap_{m=n}^{\infty} \Lambda_{m}^{c}\right\} \\
& =1-\lim _{n \rightarrow \infty} \mathscr{M}\left\{\bigcap_{m=n}^{\infty} \Lambda_{m}^{c}\right\} \\
& =1-\lim _{n \rightarrow \infty} \bigwedge_{m=n}^{\infty} \mathscr{M}\left\{\Lambda_{m}^{c}\right\}=1-0=1 .
\end{aligned}
$$

Let $\left\{X_{n}, n \geq 1\right\}$ be a sequence of independent uncertain variables, and let $\Lambda_{n}=\left\{\left|X_{n}\right|>\varepsilon\right\}, n \geq 1, \varepsilon>0$. Then $\omega \in$ $\liminf _{n \rightarrow \infty} \Lambda_{n}^{c}$ means that $\omega$ is such that $\left|X_{n}(\omega)\right| \leq \varepsilon$ for all sufficiently large $n$, and $\omega \in \lim \sup _{n \rightarrow \infty} \Lambda_{n}$ means that $\omega$ is such that there exsit arbitrarily large values of $n$ such that $\left|X_{n}(\omega)\right|>\varepsilon$. In particular, every $\omega$ for which $X_{n}(\omega) \rightarrow 0$ as 
$n \rightarrow \infty$ must be such that, for any $\varepsilon>0$, only finitely many of the real numbers $X_{n}(\omega)$ exceed $\varepsilon$ in absolute value. Hence,

$$
\begin{gathered}
\mathscr{M}\left\{\omega: \lim _{n \rightarrow \infty} X_{n}(\omega)=0\right\}=1 \Longleftrightarrow \\
\mathscr{M}\left\{\left|X_{n}\right|>\varepsilon \text { i.o. }\right\}=0
\end{gathered}
$$

for all $\varepsilon>0$. That means that

$$
\begin{gathered}
X_{n} \stackrel{\text { a.s. }}{\longrightarrow} 0 \text { as } n \longrightarrow \infty \\
\mathscr{M}\left\{\left|X_{n}\right|>\varepsilon \text { i.o. }\right\}=0
\end{gathered}
$$

for all $\varepsilon>0$. Theorem 17 tells us that if

$$
\sum_{n=1}^{\infty} \mathscr{M}\left\{\left|X_{n}\right|>\varepsilon\right\}<\infty
$$

then

$$
X_{n} \stackrel{\text { a.s. }}{\longrightarrow} 0 \text { as } n \longrightarrow \infty \text {. }
$$

Corollary 18. Let $\left\{X_{n}, n \geq 1\right\}$ be a sequence of uncertain variables, and $X$ is an uncertain variable. Let $\delta_{n}$ be a positive sequence such that $\delta_{n} \rightarrow 0$. Let $f: \mathfrak{R}_{+} \rightarrow \mathfrak{R}_{+}$be a continuous function such that $0=f(0)<f(x) \leq f(y)$ for all $0<x<y$. If one of the following conditions is satisfied

$$
\begin{aligned}
& \text { (a) } \sum_{n=1}^{\infty} \mathscr{M}\left\{\left|X_{n}-X\right|>\varepsilon\right\}<\infty, \quad \forall \varepsilon>0, \\
& \text { (b) } \sum_{n=1}^{\infty} E f\left(\left|X_{n}-X\right|\right)<\infty \\
& \text { (c) } \sum_{n=1}^{\infty} \mathscr{M}\left\{\left|X_{n}-X\right|>\delta_{n}\right\}<\infty
\end{aligned}
$$

then

$$
X_{n} \stackrel{\text { a.s. }}{\longrightarrow} X \text {. }
$$

Proof. The results follow by setting in Theorem $17 \Lambda_{n}=$ $\left\{\left|X_{n}-X\right|>\varepsilon\right\}$ for (a) and, respectively, $\Lambda_{n}=\left\{\left|X_{n}-X\right|>\delta_{n}\right\}$ for (c). The result from (b) follows by the inequality

$$
\mathscr{M}\left\{\left|X_{n}-X\right| \geq \varepsilon\right\} \leq \frac{E f\left(\left|X_{n}-X\right|\right)}{f(\varepsilon)} .
$$

\section{Conclusion}

In this paper, within the framework of uncertainty theory, we investigated the problem of in what situations the uncertain measure of an event can only be zero or one and derived the zero-one laws in uncertainty theory. Some results of almost sure convergence of uncertain sequence were presented.

\section{Conflict of Interests}

The authors declare that there is no conflict of interests regarding the publication of this paper.

\section{References}

[1] B. Liu, "Some research problems in uncertainty theory," Journal of Uncertain Systems, vol. 3, no. 1, pp. 3-10, 2009.

[2] B. Liu, Uncertainty Theory, Springer, Berlin, Germany, 2nd edition, 2007.

[3] X. W. Chen and B. Liu, "Existence and uniqueness theorem for uncertain differential equations," Fuzzy Optimization and Decision Making, vol. 9, no. 1, pp. 69-81, 2010.

[4] K. Yao, J. Gao, and Y. Gao, "Some stability theorems of uncertain differential equation," Fuzzy Optimization and Decision Making, vol. 12, no. 1, pp. 3-13, 2013.

[5] K. Yao and X. W. Chen, "A numerical method for solving uncertain differential equations," Journal of Intelligent \& Fuzzy Systems, vol. 25, no. 3, pp. 825-832, 2013.

[6] B. Liu, Uncertainty Theory, Springer, Berlin, Germany, 4th edition, 2015.

[7] X. W. Chen, "American option pricing formula for uncertain financial market," International Journal of Operations Research, vol. 8, no. 2, pp. 32-37, 2011.

[8] J. J. Sun and X. W. Chen, "Asian option pricing formula for uncertain financial market," Journal of Uncertainty Analysis and Applications, vol. 3, article 11, 2015.

[9] Z. Q. Zhang and W. Q. Liu, "Geometric average Asian option pricing for uncertain financial market," Journal of Uncertain Systems, vol. 8, no. 4, pp. 317-320, 2014.

[10] X. W. Chen and J. Gao, "Uncertain term structure model of interest rate," Soft Computing, vol. 17, no. 4, pp. 597-604, 2013.

[11] Z. Q. Zhang, D. A. Ralescu, and W. Q. Liu, "Valuation of interest rate ceiling and floor in uncertain financial market," Fuzzy Optimization and Decision Making, pp. 1-16, 2015.

[12] Y. H. Liu, X. W. Chen, and D. A. Ralescu, "Uncertain currency model and currency option pricing," International Journal of Intelligent Systems, vol. 30, no. 1, pp. 40-51, 2014.

[13] B. Liu and K. Yao, "Uncertain multilevel programming: algorithm and applications," Computers \& Industrial Engineering, vol. 89, pp. 235-240, 2015.

[14] Y. H. Liu and D. A. Ralescu, "Risk index in uncertain random risk analysis," International Journal of Uncertainty, Fuzziness \& Knowledge-Based Systems, vol. 22, no. 4, pp. 491-504, 2014.

[15] Y. Zhu, "Uncertain optimal control with application to a portfolio selection model," Cybernetics and Systems, vol. 41, no. 7, pp. 535-547, 2010.

[16] X. Yang and J. Gao, "Uncertain differential games with application to capitalism," Journal of Uncertainty Analysis and Applications, vol. 1, article 17, 2013.

[17] X. Gao, "Some properties of continuous uncertain measure," International Journal of Uncertainty, Fuzziness \& KnowledgeBased Systems, vol. 17, no. 3, pp. 419-426, 2009.

[18] B. Liu, Uncertainty Theory: A Branch of Mathematics for Modeling Human Uncertainty, Springer, Berlin, Germany, 2010. 


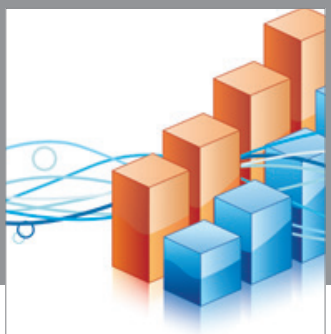

Advances in

Operations Research

vatem alat4

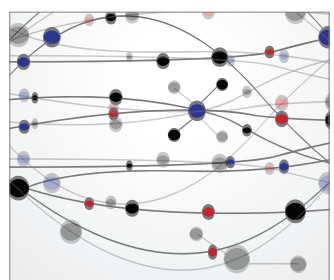

\section{The Scientific} World Journal
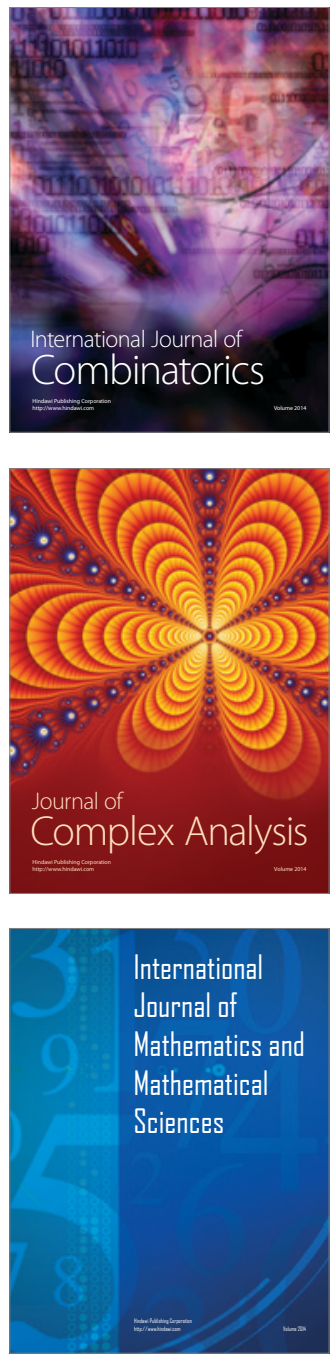
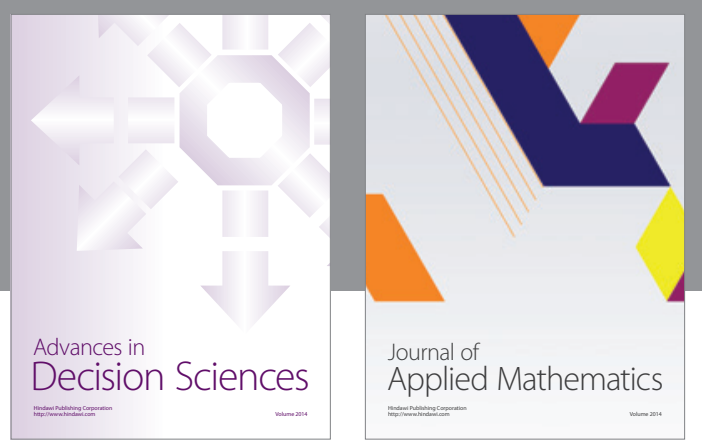

Algebra

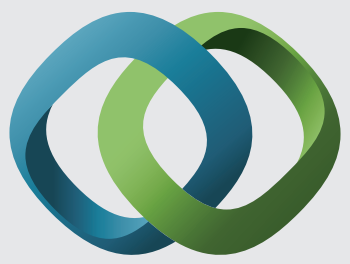

\section{Hindawi}

Submit your manuscripts at

http://www.hindawi.com
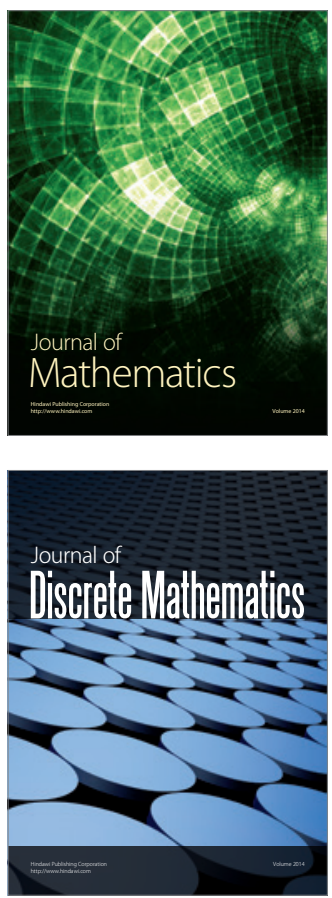

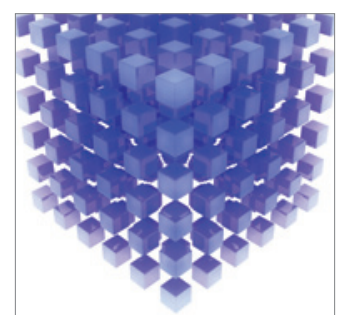

Mathematical Problems in Engineering
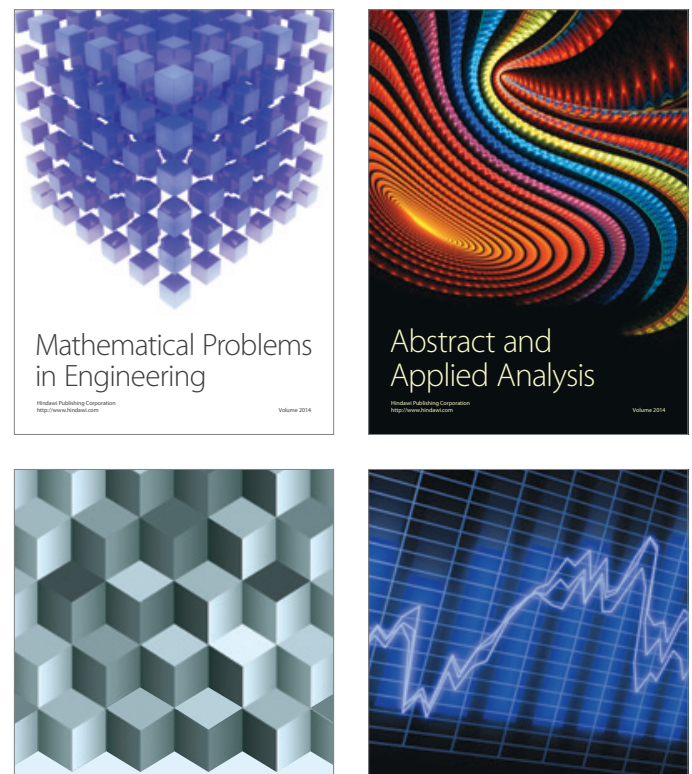

Journal of

Function Spaces

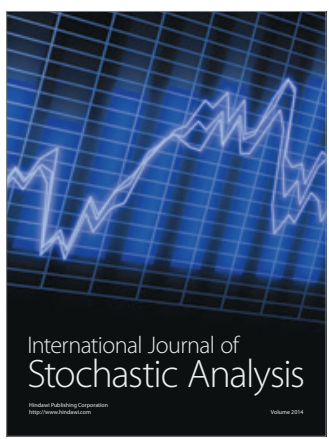

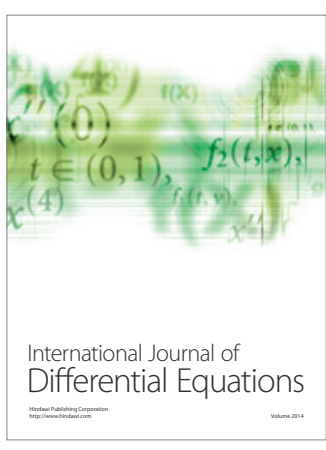
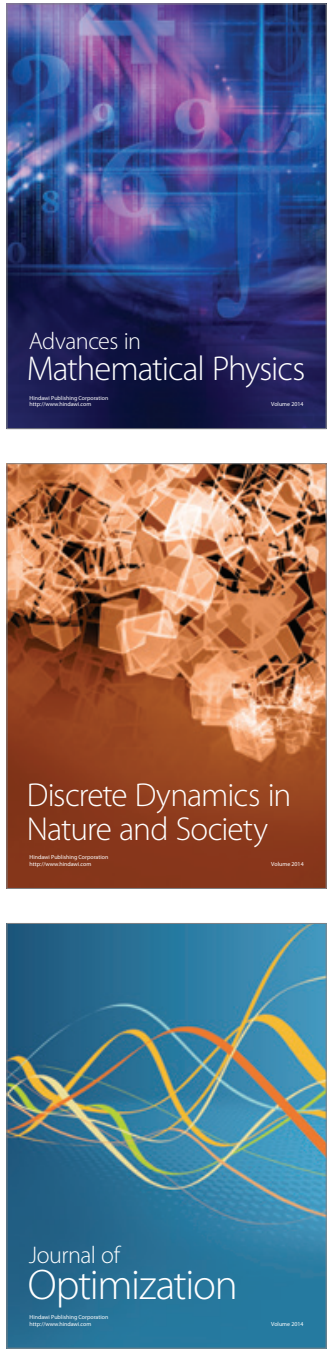